\title{
Effect of different terminators on transcription regulatory factor CIrB and XInR in Penicillium oxalicum 114-2
}

\author{
Qin Yan ${ }^{1, \mathrm{a}}$, YananWang ${ }^{1, \mathrm{~b}}$, Xiaoming Bao ${ }^{1, \mathrm{c}}$, Yinbo $\mathrm{Qu}^{2, \mathrm{~d}}$, XinLi Liu ${ }^{1, \mathrm{e}}$, \\ Zhonghai $\mathrm{Li}^{*}, \mathrm{f}$
}

\author{
${ }^{1}$ Shandong Provincial Key Laboratory of Microbial Engineering, Department of Bioengineering, Qi \\ Lu University of Technology, Jinan 250353, China \\ ${ }^{2}$ State Key Laboratory of Microbial Technology, Shandong University, Jinan 250100, China \\ a18363004106@163.com, 'bangya nan2013@163.com, ${ }^{\mathrm{b}}$ bxm@sdu.edu.cn, \\ dquyinbo@sdu.edu.cn, ${ }^{\mathrm{e}}$ vip.|x|@163.com, ${ }^{\mathrm{f}}$ Izhlzh@vip.126.com
}

* Correspondence: vip.|x|@163.com, Izhlzh@vip.126.com; Tel \& fax: +86 53189631776

\begin{abstract}
Keywords: Lignocellulose Cellulase and hemicellulase Terminators CIrB and XInR
Abstract. ClrB and XlnR positively regulated cellulase and hemicellulase expression in Penicillium oxalicum 114-2. In addition,terminators, as promoters, play an important role in gene transcription. $\mathrm{T}$ - his research mainly investigated the effects of TbglII, TcbhI, TegI, TD, TgpdA on the regulatory fact- ors ClrB and XlnR. By replacing TclrB and TxlnR with TbglII, TcbhI, TegI, TD, TgpdA, we explored the effect of different terminators on two regulatory factors and ultimately achieved the purpose of improving cellulase activity. The results showed that the terminator had a significant influence on the regulatory function of regulators, and the FPA, CMC, $p$ NPX and $p$ NPC enzyme activity of the recom- binant strains obtained were significantly higher than that of wild-type 114-2 and the highest FPA enzyme activity reached $1.4 \mathrm{U} / \mathrm{mL}(\mathrm{c}-\mathrm{d} 15-2-1)$. These were clearly not enough compared to high-yield cellulases strains, but here provided new insight into the improving the cellulase and hemicellulase activity. Terminators had a huge potential for the production of cellulase and hemicellulase.
\end{abstract}

\section{Introduction}

At present, it was undeniable that oil is the most important and indispensable resource in the world. Oil and petrochemical products are closely related to the basic necessities of life. However, oil is a nonrenewable resource. With its gradual depletion, oil reserves are getting less and less, and the environment was also destroyed. Therefore, finding a renewable, green energy is imminent.

Lignocellulose was the most abundant biomass on the earth. Lignocelluloses are mainly found in plant cell walls. It can be degraded into fermentable sugar by enzymatic hydrolysis, which are used in the production of biomass-based biorefineries ${ }^{[1]}$. Millions of years of evolution, different microorganisms are also in their own development of their own unique enzyme mechanism of degradation. Cellulase from filamentous fungi (Trichoderma, Aspergillus and Penicillium) is extracellular free enzyme, which is relatively simple. So filamentous fungi are the major source of cellulase ${ }^{[2,3]}$ and they have always been the focus of cellulase research. In this study, Penicillium oxalicum 114-2 was also used as a starting strain to study its cellulolytic enzymes.

Penicillium has a complete cellulolytic and hemicellulolytic enzyme system and Potential for bioenergy ${ }^{[4,5]}$ that provided a good platform for the degradation of cellulose. Besides the lignocelluly- tic enzyme system of P.oxalicum is more diverse conpared with the main industrial strain T. reesei ${ }^{[6]}$. It is reported that the degradation intensity of cellulase is depends on the transcription regulatory factor. ClrB is a global positive regulator, and ClrB/CLR -2, is essential for cellulase expression in $P$. oxalicum, N.crassa and Aspergillus nidulans ${ }^{[7,8]}$. It also belongs to the zinc finger protein transcription factor superfamily. ClrB of Penicillium oxalicum and homologous of other filamentous fungi(Clr-2 in N. crassa, ClrB in A. nidlans, and ManR in A. oryzae) recent studies have identified that it can promote the major cellulase genes and several hemicellulase genes expression, but no significant effect on the expression of xylanase gene. XlnR protein in Aspergillus niger and 
Trichoderma reesei is the first transcriptional regulatory activator found to play a regulator role in the expression of cellulase and hemicellulase ${ }^{[9,10]}$. XlnR contains the Zn2Cys6-type zinc finger binding domain in fungi. In the xylanase and multiple cellulases expression has a crucial re-gulatory role.

$\mathrm{ClrB}, \mathrm{XlnR}$ on the production of cellulase is self-evident. But most researchers will focus on the rep- lacement of the stronger promoter, overexpression, and directional transformation ${ }^{[11,12]}$. Few resea-rchers have studied the effects of terminators on cellulase production. Terminator, like promoters, is essential elements of genes and have a significant impact on gene transcription. In this study, we focused on the effect of different terminators on the activity of major cellulase enzymes in 114-2. The main choice is the termination of highly expressed cellulase genes in filamentous fungi and the termination of the genes with strong promoters.

\section{Materials and methods}

Strains and culture media. Penicillium oxalicum 114-2 provided by yinbo Qu professor in ShanDong University. Other strains were constructed in this study. All strains used were shown in (Table 1).

P.oxalicum was cultivated on wheat straw bevel for conidia culture at $30{ }^{\circ} \mathrm{C}$. After three days, conidia inoculated into glucose medium at a concentration of $10^{7}$ per $\mathrm{mL}$ and grown at $30^{\circ} \mathrm{C}, 200 \mathrm{rpm}$ for $20 \mathrm{~h}$. Then sterile filtration and weighed $0.5 \mathrm{~g}$ mycelium inoculated into fermentation medium, $30{ }^{\circ} \mathrm{C}, 200 \mathrm{rpm}$ for 6 days used in enzyme avtivity assays.

All medium preparation methods: wheat straw bevel: $100 \mathrm{~g}$ wheat straw boiled in $1 \mathrm{~L}$ tap water for 30 mins, then 8 layers of gauze filter, the filtrate volume to $1 \mathrm{~L}$, added 20 g agar. Glucose medium: 20 $\mathrm{g} / \mathrm{L}$ glucose, $20 \mathrm{~mL} / \mathrm{L}$ vogel's salt. Fermentation medium: $0.6 \%$ avice, $2 \%$ corn cob residue, $4.6571 \%$ wheat bran, $1 \%$ soybean cake power, $0.2 \%\left(\mathrm{NH}_{4}\right)_{2} \mathrm{SO}_{4}, 0.2789 \% \mathrm{NaNO}_{3}, 0.1 \%$ Urea, $0.3 \% \mathrm{KH}_{2} \mathrm{PO}_{3}$ and $0.05 \% \mathrm{MgSO}_{4} \cdot 7 \mathrm{H}_{2} \mathrm{O}$.

Construction of recombinant P.oxalicum strains: The target cassettes were constructed by doub- le-jointg $\mathrm{PCR}^{[13]}$. For deletion TClrB and TXlnR, the up and down homologous arm and TbglII, TegI TcbhI, TD were amplified form the genomic DNA of 114-2, the TgpdA was amplified form the geno- mic DNA of Aspergillus nidulans. The marker genes was hygromycinB $(h p h)$. The cassettes were then obtained by nest-PCR.Final, the cassettes were transformed into P.oxalicum114-2, respectively. Bec- ause the upstream/down homology arm is sited in $\mathrm{T} c \operatorname{lr} B$ or $\mathrm{T} x \ln R$ sequence upstream/downstreamt. TclrB or TxlnR was replaced by TbglII, TcbhI, TegI, TD, TgpdA and hph according to the principle of homologous recombination. The construction of the target cassette was shown in ( Fig. 1). All the pri- mers used in this study were shown in (Table 2).

Enzyme assays: In this study, FPase, CMCase, $p$ NPCase, $p$ NPXase were tested at 3, 4, 5, 6 day in the fermentation process. Samples were centrifuged at $4{ }^{\circ} \mathrm{C}$ and $12000 \mathrm{rpm}$ for10mins, then transformd the supernatant into a new centrifuge tube and placed on ice until ready for use. FPase, CMCase measured by DNS method. Taken $0.1 \mathrm{~g}$ Whatman filter paper into the bottom of the glass tube, add $1.5 \mathrm{~mL}$ HAC-NaAC buffer $(\mathrm{pH} \mathrm{4.8)}$ ) and $500 \mu \mathrm{L}$ (diluted to the appropriate range) crude enzyme solution, mix well and incubated at $50{ }^{\circ} \mathrm{C}$ for $1 \mathrm{~h}$. Then adding $3 \mathrm{~mL}$ DNS $(20.8 \mathrm{~g} / \mathrm{L} \mathrm{NaOH}$, $6 \mathrm{~g} / \mathrm{L} \mathrm{DNS}, 6 \mathrm{~g} / \mathrm{L}$ seignette salt, $5 \mathrm{~g} / \mathrm{L} \mathrm{Na}_{2} \mathrm{SO}_{3}, 5 \mathrm{~g} / \mathrm{L}$ redistilled phenol) termination of the reaction, boiled 10 mins, added distilled water to a constant volume of $25 \mathrm{~mL}$ when the liquid cooled. Mix well and at a wavelength of $540 \mathrm{~nm}$ determ- ination the OD value. The CMCase, in addition to the reaction substrate was changed to $1 \%$ sodium carboxymethyl cellulose and water bath $30 \mathrm{mins}$, The remaining conditions were samed with the FPA enzyme assay, and the released gluose was determined at 540 $\mathrm{nm}$. Take 100ul (diluted to the appropri- ate range) crude enzyme solution, added 50ul substrate $p$-nitrophenyl- $\beta$-D-cellobioside $(p \mathrm{NPC}$ ) or $p$ nitrophenyl- $\beta$-D-xylopyranoside $(p \mathrm{NPX})(1 \mathrm{mg} / \mathrm{ml})$, mix well, $50{ }^{\circ} \mathrm{C}$ for 30 mins. The reaction was stoped with the addition of $150 \mu \mathrm{L} 10 \% \mathrm{NaCO}_{3}(\mathrm{w} / \mathrm{v})$, and the released $p$ nitrophenol was determined at $420 \mathrm{~nm}$. One unit of all enzyme activities was defined as 
the amount of enzyme that liberated $1 \mu \mathrm{mol}$ of product (glucose equivalents or $p$-nitrophenol) per minute under the assay conditions used.

Table 1 All strains in this study

\begin{tabular}{ccc}
\hline Strain & Source & Transformation way \\
\hline $114-2$ & provided by yinbo Qu professor & wild-type \\
$\mathrm{c}-\mathrm{d} 15-2-1$ & In research & TD replace TclrB \\
$\mathrm{c}-\mathrm{e} 7-1-1$ & In research & TegI replace TclrB \\
$\mathrm{x}-\mathrm{b} 3-1-1$ & In research & TbglII replace TxlnR \\
$\mathrm{x}-\mathrm{b} 7-1-1$ & In research & TbglII replace TxlnR \\
$\mathrm{x}-\mathrm{c} 15-1-1$ & In research & TcbhI replace TxlnR \\
$\mathrm{x}-\mathrm{d} 9-2-1$ & In research & TD replace TxlnR \\
$\mathrm{x}-\mathrm{e} 4-1-1$ & In research & TegI replace TxlnR \\
$\mathrm{x}-\mathrm{e} 9-1-1$ & In research & TegI replace TxlnR \\
$\mathrm{x}-\mathrm{g} 4-2-1$ & In research & TgpdA replace TxlnR
\end{tabular}

Table 2 All the primers used in research

\begin{tabular}{|c|c|}
\hline Primer name & Primer sequences \\
\hline Six-hph-F & GGAAGGATACAGTCGCTAGC \\
\hline Six-hph-R & CGTTCACACGTGAAGC \\
\hline Tbgl II -F & TAAATATGGGCTACGAGAGACCCGA \\
\hline Tbgl II-six-hph-R & GAGTTGCTAGCGACTGTATCCTTCCGGAGCAAGGGAAATCAAACTG \\
\hline Tcbh I -F & TAAGTCTTGAGTGGTCGTCGAGGTC \\
\hline Tcbh I-six-hph-R & GAGTTGCTAGCGACTGTATCCTTCCAATGGATGGCAAGGTTCCAG \\
\hline TD-F & TAAACGAAAAAGTTAAAAGGGAAAG \\
\hline TD-six-hph-R & GAGTTGCTAGCGACTGTATCCTTCCTCTTTGACGCGACGGACGT \\
\hline Teg I-F & TGATTCAAATTGAATGGAGGGGAAT \\
\hline Teg I-six-hph-R & GAGTTGCTAGCGACTGTATCCTTCCACCAAAAGATTCAGGTCATGT \\
\hline TGpdA-F & TAGGAAACAGGTCGGAAGCCAATGGC \\
\hline TGpdA-six-hph-R & GAGTTGCTAGCGACTGTATCCTTCCTCCTCATCATCTACCATTGTCG \\
\hline (qt)TclrB U-F & GGAGGCTGGTACTGCCACTACATC \\
\hline clrB-Six-hph-D-F & CTGCGGCCGCTTCACGTGTGAACGCGGGAGGTGAGCTACTTGCCT \\
\hline (qt)clrB-D-R & GGTTTATGGAACTCACCTGACTTG \\
\hline TclrB U-F & TGCTGAATATCGTCCTTGCACTCG \\
\hline clrB-D-R & AACGACGATGGCCTTACCTG \\
\hline (qt) $\mathrm{T} x \ln R-\mathrm{U}-\mathrm{F}$ & TCGGCGAGCGAATACAAGGCT \\
\hline TxlnR-Six-hph-D-F & CTGCGGCCGCTTCACGTGTGAACGCATGAATTTAGCCATGCAGGT \\
\hline (qt) $T \times \ln R$ D-R & CGCCACGAAAGAACTCCAACTC \\
\hline TxlnR-U-F & CCGAGGGCGTGTTTGCCAGA \\
\hline TXlnR D-R & GGCAGCATTGGCAAGAGTG \\
\hline TclrB-Tbgl II-U-R & TCGGGTCTCTCGTAGCCCATATTTACTGGTAAAATGCAGGGGGGTTCGC \\
\hline TclrB-TD-U-R & СТTTСССТTTTAACTTTTTCGTTTACTGGTAAAATGCAGGGGGGTTCGC \\
\hline TClrB-Teg I-U-R & АTTCСССТССАТTCAATTTGAATCACTGGTAAAATGCAGGGGGGTTCGC \\
\hline TclrB-TGpdA-U-R & CCATTGGCTTCCGACCTGTTTCCTACTGGTAAAATGCAGGGGGGTTCGC \\
\hline TClrB-Tcbh I-U-R & GACCTCGACGACCACTCAAGACTTACTGGTAAAATGCAGGGGGGTTCGC \\
\hline TxlnR-Tbgl II-U-R & CTTCGGGTCTCTCGTAGCCCATATTTACAGTGCAAGGCCGCTGCCGTCGCC \\
\hline TxlnR-Tcbh I-U-R & GACCTCGACGACCACTCAAGACTTACAGTGCAAGGCCGCTGCCGTCGCC \\
\hline TxlnR-TD-U-R & СTTTCССТTTTAACTTTTTCGTTTACAGTGCAAGGCCGCTGCCGTCGCC \\
\hline TxlnR-Teg I-U-R & ATTCCССТCСАTTCAATTTGAATCACAGTGCAAGGCCGCTGCCGTCGCC \\
\hline TxlnR-GpdA-U-R & GCCATTGGCTTCCGACCTGTTTCCTACAGTGCAAGGCCGCTGCCGTCGCC \\
\hline
\end{tabular}

\section{Results}


The choice of terminator and recombinant P.oxalicum strains: Based on the reported and transcriptional analysis of Penicillium oxalicum, we selected the terminators of $b g l 1, \operatorname{cbh} 1, D$, eg 1 and gpda genes. After PCR validation, each expression cassette had the correct recombinant strain.

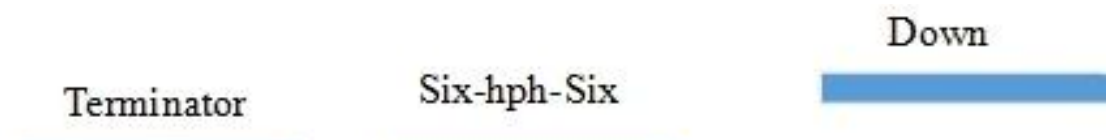

Fusion PCR

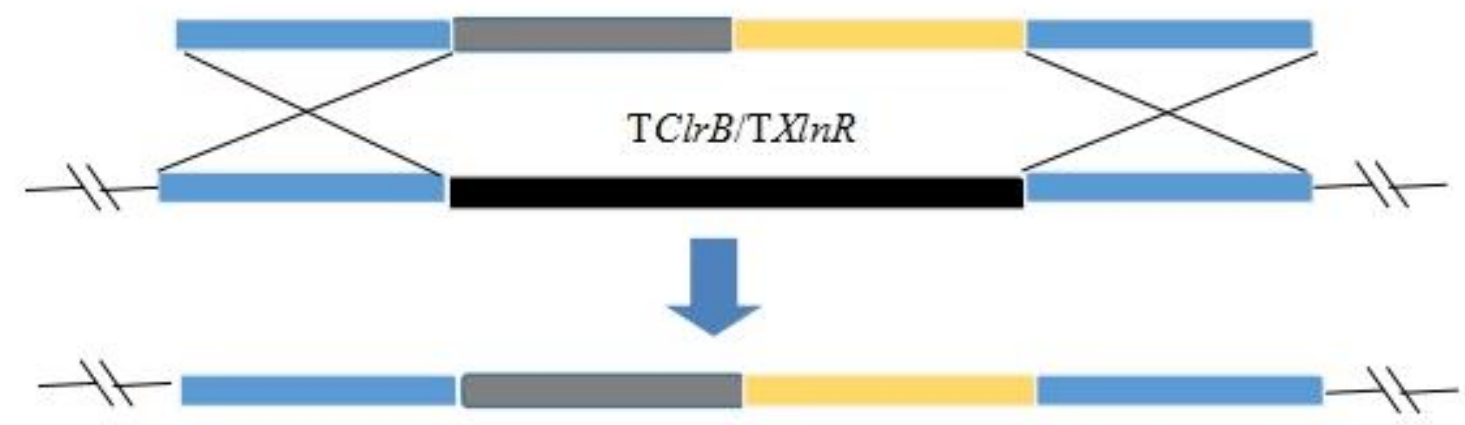

Fig.1.Expression cassette construction and transformation diagram.Terminators including TbglII,TcbhI,TD,TegI,TgpdA.
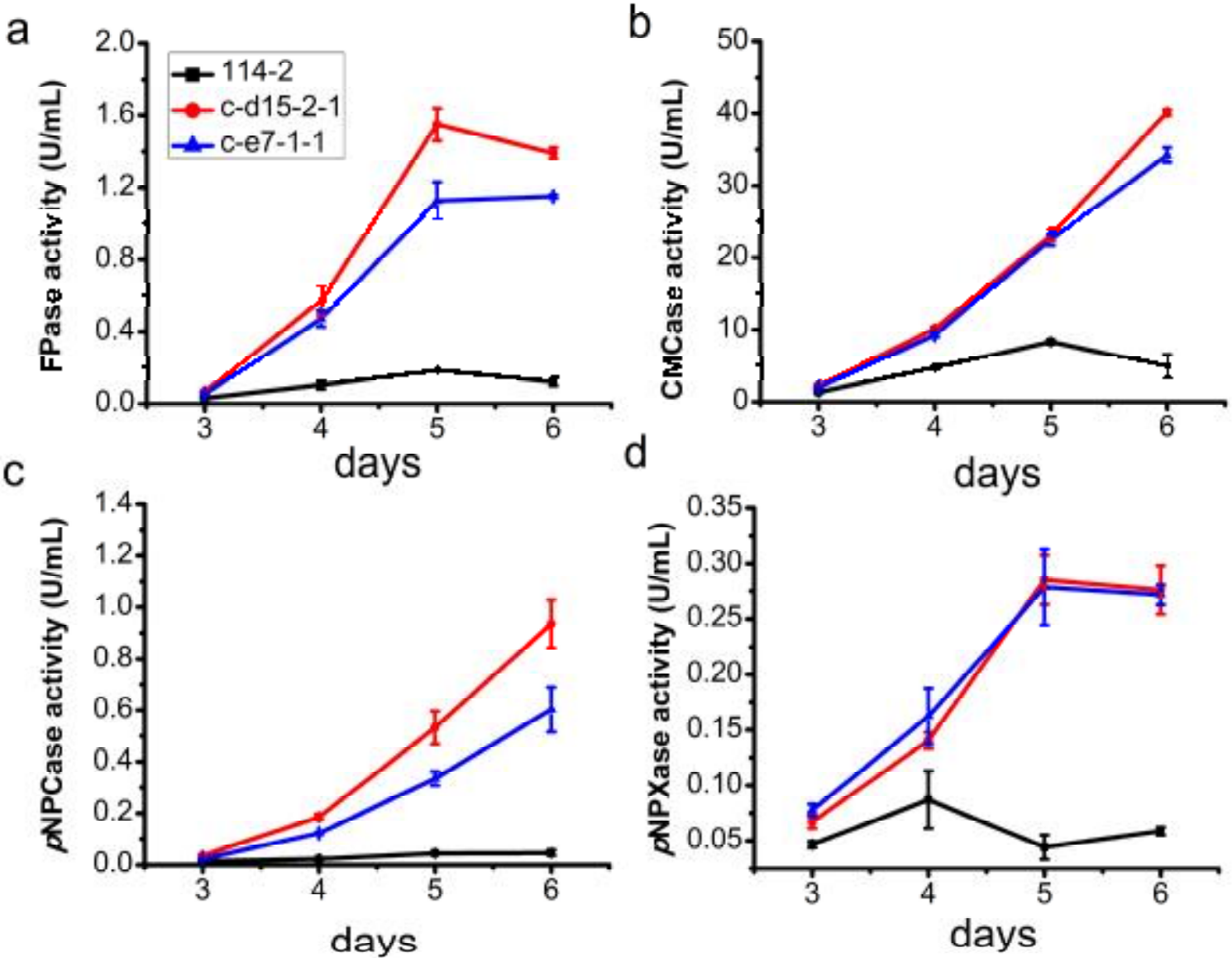
Fig. 2 Enzyme activity analyse of 114-2 and different terminators replace the $\operatorname{clr} B$ terminator recombinant strains.(a) FPase (b) CMCase (c) $p$ NPCase (d) $p$ NPXase
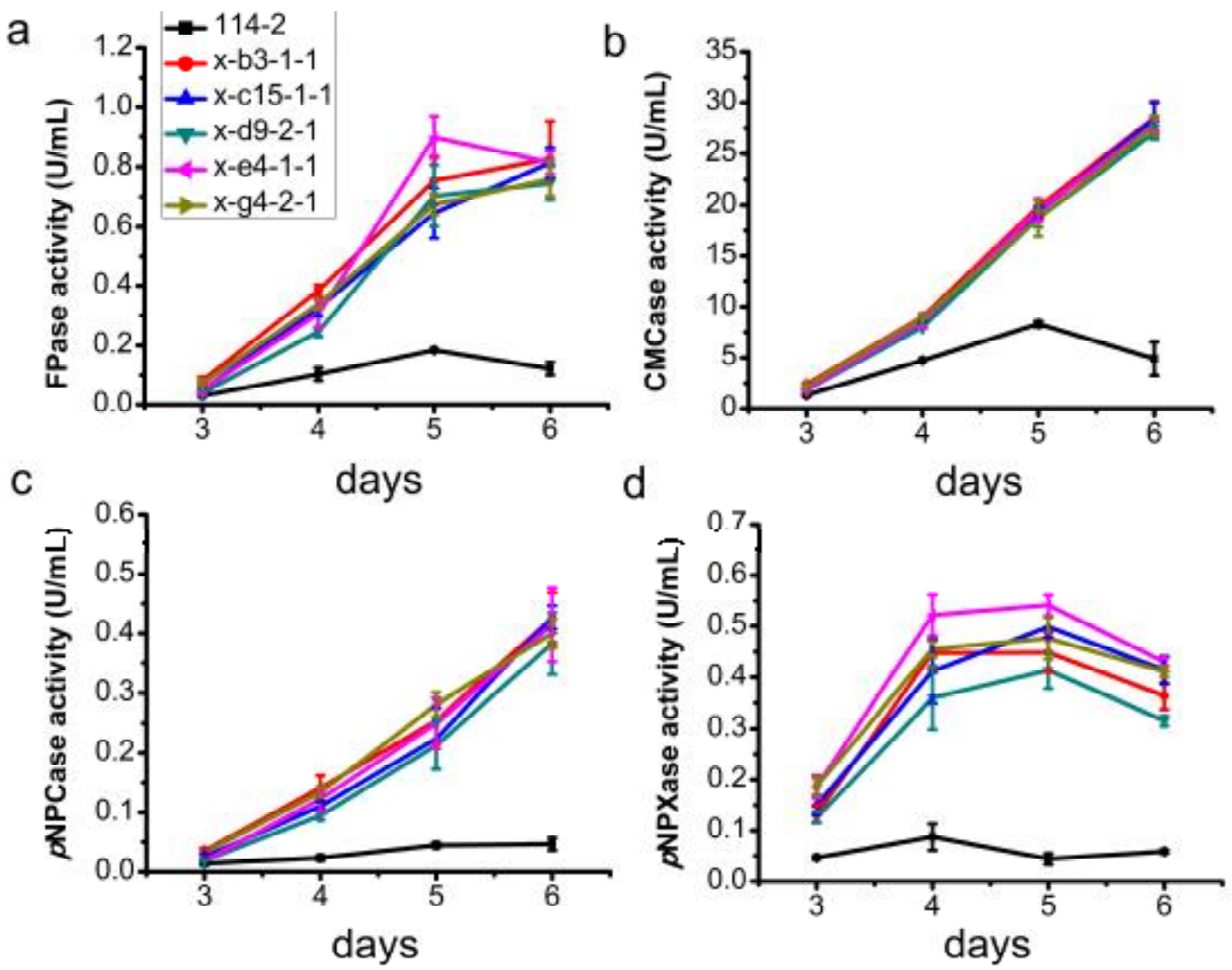

Fig. 3 Enzyme activity analyse of 114-2 and different terminators replace the $x \ln R$ terminator recombinant strains.(a) FPase (b) CMCase (c) $p$ NPCase (d) $p$ NPXase

Effect of terminator on ClrB regulatory function: Transcriptional regulator ClrB regulates the transcriptional expression of cellulase and most hemicellulose genes and it was a global regulator. In the present study, the terminator region of $\mathrm{ClrB}$ was replaced by different terminator regions. It was found that TD and TegI were significantly enhanced the enzyme of FPA, CMC, $p$ NPC and $p$ NPX (Fi g.2) but Tbgl, TcbhI and TgpdA had no significant effect on enzyme activity (data not shown). All the ClrB recombinant trains, the FPA enzyme activity appeared the maximum on the fifth day of the fermentation. The c-d15-2-1 (TD replacement TclrB) strain reached 1.4 U/mL, c-e7-1-1-1 (TegI replacement $\operatorname{Tc}(r B$ ) also reached $1.21 \mathrm{U} / \mathrm{mL}$, which was 7.8 and 6.7 fold than that of 114-2, respectively. The c-d15-2-1 was slightly better than c-e7-1-1-1 strain. In the other three enzyme activities, the recombinant strain was still on the rise in the sixth day, while 114-2 had no increasing trend on the sixth day. Compared with 114-2, the c-d15-2-1strain on CMC, $p$ NPC, $p$ NPX enzyme activity increased by5-fold, 20-fold, 4.5-fold, respectively. And c-e7-1-1 increased by 4-fold, 12-fold, 4.5fold (Fig. 2).

However, the c-d15-2-1 strain had a higher enzyme activity than the c-e 7-1-1-1 strain. It is possible that the effect of the $\mathrm{T} D$ on the $\operatorname{clr} B$ gene expression was more pronounced.

Effect of terminator on $X \ln R$ regulatory function: The transcription regulation factor XlnR mainly regulates the expression of hemicellulase and partial cellulase genes. In this study, the selected terminators in the research had a significant effect on the $x \ln R$ gene experess (Fig.3). Recombinant strains at FPA, CMC, $p$ NPC, $p$ NPX enzyme activity was 4.5-fold, 3.4 -fold, 9-fold, 10-fold compared with 114-2, respectively. Although there was a significant difference between the recombinant strains and wild-tyupe 114-2, howevre, the difference between recombinant strains was 
not obvious. It was possible that these terminators had the same degree of influence on the $x \ln R$ gene. Interestingly, the FPA, CMC, and $p N P C$ enzyme activity that replaced the TclrB perform better than those that replaced the T $x \ln R$, but $p$ NPX enzyme activity was just on the opposite. This may be because XlnR mainly regulates the hemicellulase gene (xylanase), while ClrB mainly regulates the expression of the cellulase gene ( $c b h I, e g I)$, leading to the above results.

The study also found that the five selected terminators in the experiment had a significant effect on $x \ln R$, but only two terminators on the $\operatorname{clr} B$ gene. It is suggested that $x \ln R$ may be more susceptible to the changed by the terminator than the $\operatorname{cr} B$ gene, but further study was needed.

\section{Discussion}

The transcription regulation factor of $\mathrm{ClrB}$ and $\mathrm{X} \ln \mathrm{R}$ had an important impact on the cellulase and $\mathrm{h}$ emicellulase yield. Terminators also had an important effect on gene transcription. In this study, the terminators of $c \operatorname{lr} B$ and $x \ln R$ were replaced by different terminators to study the role of terminator on gene transcription. It was obvious that terminators had a significant impact on gene expression, indicating that the modification of the terminator can be used as a new method for the strain transformation.

However, the same terminator had different effects on different genes, TD and TegI had better effect on $\operatorname{clr} B$ gene than that of $x \ln R$ gene (Fig. 2, Fig. 3) .TcbhI, TbglII and TgpdA had no obvious effect on ClrB (results not shown), but had a significant impact on XlnR (Fig. 3). At the same time, different terminators may have similar effects on the same gene. The results of the four enzymes shown that the effects of TD, TegI, TcbhI, TbglII and TgpdA on $x \ln R$ gene are similar. Although they all had a significant effects compared to their own terminator, while there were not much difference between these terminators.

By changing the terminator region of the regulatory factor, cellulase activity was significantly increased and the highest FPA enzyme activity reached $1.4 \mathrm{U} / \mathrm{mL}$ (c-d15-2-1), although there was a gap between this and the high-yielding cellulase strain RE- $8, \mathrm{RE}-10^{[14,15]}$ however, it provided a new $\mathrm{m}$ ethod to transform strains, terminator in the transformation of strains still haved a great potential. In order to obtain high yield cellulase strains, these efforts were clearly not enough. More terminators should be screened for genetic manipulation in strains with high cellulase activity. This was the key work of our next priority.

\section{Acknowledgements}

This work was financially supported by the National Natural Science Fund of China(No.31670079)

\section{References}

[1] Kamm B, Kamm M. Biorefineries - Multi Product Processes[J]. Adv Biochem Eng Biotechnol, 2007, 105:175-204.

[2] Ferreira J A, Mahboubi A, Lennartsson P R, et al. Waste biorefineries using filamentous ascomycetes fungi: Present status and future prospects[J]. Bioresource Technology, 2016, 215:334-345.

[3] Gusakov A V. Alternatives to Trichoderma reesei, in biofuel production[J]. Trends in Biotechnology, 2011, 29(9):419-25.

[4] Liu G, Zhang L, Wei X, et al. Genomic and secretomic analyses reveal unique features of the lignocellulolytic enzyme system of Penicillium decumbens.[J]. Plos One, 2013, 8(2):e55185.

[5] Gusakov A V, Sinitsyn A P. Cellulases from Penicillium species for producing fuels from biomass.[J]. Biofuels, 2012, 3(4):463-477. 
[6] Kubicek C P, Mikus M, Schuster A, et al. Metabolic engineering strategies for the improvement of cellulase production by Hypocrea jecorina[J]. Biotechnology for biofuels, 2009, 2(1):19.

[7] Li, Z., Yao, G., Wu, R., Gao, L., Kan, Q., Liu, M., Yang, P., Liu, G., Qin, Y., Song, X., Zhong, Y., Fang, X., Qu, Y.Synergistic and dose-controlled regulation of cellulase gene expression in Penicillium oxalicum. PLoS Genet. 2015,11 (9):e1005509

[8] Coradetti S T, Glass N L. Conserved and essential transcription factors for cellulase gene expression in ascomycete fungi[J]. Proceedings of the National Academy of Sciences of the United States of America, 2012, 109(19):7397-402.

[9] Furukawa T, Shida Y, Kitagami N, et al. Identification of specific binding sites for XYR1, a transcriptional activator of cellulolytic and xylanolytic genes in Trichoderma reesei[J]. Fungal Genetics \& Biology, 2009, 46(8):564-574.

[10] van Peij N N, Visser J, de Graaff L H. Isolation and analysis of xlnR, encoding a transcriptional activator co-ordinating xylanolytic expression in Aspergillus niger.[J]. Molecular Microbiology, 1998, 27(1):131-42.

[11] Derntl C, Gudynaitesavitch L, Calixte S, et al. Mutation of the Xylanase regulator 1 causes a glucose blind hydrolase expressing phenotype in industrially used Trichoderma strains.[J]. Biotechnol Biofuels. 2013, 6(1):62.

[12] Craig J P, Coradetti S T, Starr T L, et al. Direct Target Network of the Neurospora crassa Plant Cell Wall Deconstruction Regulators CLR-1, CLR-2, and XLR-1.[J]. Mbio, 2015, 6(5):e01452.

[13] Yu J H, Hamari Z, Han K H, et al. Double-joint PCR: a PCR-based molecular tool for gene manipulations in filamentous fungi.[J]. Fungal Genetics \& Biology, 2004, 41(11):973-981.

[14] Gao L, Li Z, Xia C, et al. Combining manipulation of transcription factors and overexpression of the target genes to enhance lignocellulolytic enzyme production in Penicillium oxalicum[J]. Biotechnology for Biofuels, 2017, 10(1):100.

[15] Yao G, Li Z, Gao L, et al. Redesigning the regulatory pathway to enhance cellulase production in Penicillium oxalicum.[J]. Biotechnology for Biofuels, 2015, 8(1):71. 